\title{
XVIII Congrès de la Société d'Andrologie de Langue Française
}

\author{
Montpellier \\ 13-14-15 Décembre 2001
}

\section{Estimation du taux d'aneuploïdies des spermato- zoïdes épididymaires prélevés en vue d'icsi chez des hommes à caryotype normal \\ C. TRIPOGNEY, C. ROUX, O. BLAGOSKLONOVA, F. FELLMANN, H. BITTARD, J.L. BRESSON}

Service de Cytogénétique, Immunocytologie, Biologie du Développement et de la Reproduction - Service d'Urologie CECOS \& EA $n^{\circ} 3185$ : Génétique et Reproduction ; CHU Saint Jacques, place Saint Jacques, 25030 Besançon

\section{Introduction}

Depuis une dizaine d'année de nombreux travaux ont tenté d'estimer par FISH le taux d'aneuplö̈dies des spermatozoïdes humains éjaculés. La majorité des études réalisées chez l'homme à caryotype normal montrent que le taux de disomie par paire chromosomique se situe entre 0,15 et $0,30 \%$.

Le but de ce travail a été d'évaluer le taux d'aneuploïdies de spermatozoïdes épididymaires prélevés chirurgicalement lors de techniques MESA (Microscopical Epididymal Sperm Aspiration).

\section{Matériels et méthodes}

Les spermatozoïdes épididymaires ont été obtenus chez 5 patients présentant une azoospermie excrétoire (origine post infectieuse : 2 patients - post traumatique : 2 - malformative : 1) et pour lesquels le bilan génétique habituel (caryotype, recherche de mutation du gène CFTR et de microdélétion de l'Y) est sans particularité. Pour chaque patient, un échantillon de spermatozoïdes a été immédiatement lavé et conditionné pour l'hybridation. La technique de triple FISH X,Y,8 (X : DXZ1, Y : PHY2.1, $8:$ D8Z2) a été appliquée sur des étalements de spermatozoïdes à noyaux préalablement décondensés. Parallèlement les spermatozoïdes éjaculés de 4 hommes à sperme et caryotype normaux ont servi de témoins.

\section{Résultats}

L'analyse en FISH de 18013 spermatozoïdes épididymaires fournit les résultats suivants :

Les valeurs observées sur la population de spermatozoïdes épididymaires analysée ne diffèrent pas significativement du groupe témoin.

Des différences ponctuelles du taux de disomie pour certains chromosomes peuvent être observées chez un patient, mais elles ne sont pas statistiquement significatives.

\section{Conclusion}

L'utilisation de spermatozoïdes épididymaires en ICSI, chez des patients présentant une azoospermie excrétoire, ne semble pas présenter un risque accru de transmission de déséquilibre chromosomique à l'enfant ou de déviation du sexe ratio.

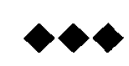

\section{Résultats préliminaires de l'enquête de la SALF concernant le dépistage des microdélétions du chromosome $\mathbf{Y}$}

\section{AKNIN-SEIFER 1,2, H. LEJEUNE 3, I. MAUBON 1,} R. LEVY1

1Laboratoire de Biologie de la Reproduction et 2Laboratoire de Génétique de l'hôpital Nord, Saint-Etienne, 42055, France, 3Département de Médecine de la Reproduction, Lyon, France

Introduction : Les microdélétions du chromosome $\mathrm{Y}$ peuvent être détectées chez les patients infertiles présentant des troubles sévères de la spermatogenèse. Ce dépistage est conseillé avant réalisation de l'ICSI. Le but de l'enquête était tout d'abord de faire "l'état des lieux " sur la pratique en France du dépistage des microdélétions du chromosome $\mathrm{Y}$ en tentant de répondre aux questions suivantes :

- Combien de praticiens et de centres d'AMP recherchent les 
microdélétions du chromosome $\mathrm{Y}$ ?

- Quelles sont les indications retenues?

- Quelles sont les méthodes employées ?

- Comment est financé le dépistage?

- Combien de détections sont effectuées chaque année en France ?

Les objectifs, à terme, étaient :

- de pouvoir discuter d'une prise en charge par les organismes d'Assurance Maladie,

- de préciser si un consensus se dégage en ce qui concerne les indications du dépistage,

- de préciser les méthodes les plus adaptées selon le rapport coût - efficacité et

- de pouvoir discuter de la mise en place d'un “ contrôle de qualité*" national.

* échantillons positifs et négatifs soumis à l'analyse dans les différents laboratoires

Matériels et Méthodes : Questionnaires: Deux questionnaires spécifiques ont été élaborés : un destiné aux prescripteurs de cette analyse et un destiné aux biologistes moléculaires réalisant cette recherche. Centres: Les questionnaires ont été adressés aux responsables (clinicien et biologiste) de chacun des 82 centres agréés en AMP.

Résultats : 81 réponses "Biologistes" ont été obtenues soit par retour de questionnaires, soit lors d'un entretien téléphonique : 30 centres font cette détection (mais seuls 20 ont effectivement à ce jour renvoyé leur formulaire), 51 ne le font pas (mais 15 formulaires seulement ont été retournés). Parmi les centres d'AMP ne faisant pas cette analyse, certains assurent le transfert vers un laboratoire effectuant cette détection : autre laboratoire d'AMP, unité de cytogénétique, ou structure polyvalente privée (laboratoires MERIEUX, CERBA ...). Les résultats préliminaires concernant la réalisation de la détection des microdélétions mettant en évidence une extrême diversité des pratiques seront développés lors du congrès.

Conclusions : La recherche des microdélétions est une analyse en plein développement mais qui suscite encore de nombreuses questions. L'évolution vers une technique standardisée, soumise à un contrôle de qualité national, ainsi que la reconnaissance de cette analyse par la Sécurité Sociale et sa cotation apparaissent comme des étapes essentielles à sa généralisation en routine.

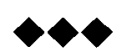

\section{Varicocèle: une approche médico-chirurgicale}

\section{F. KIRSCH-NOIR 1, BIGOT2 2 , V. IZARD1, M. TASSART2, M. BAZOT ${ }^{2}$, O. TRAXER ${ }^{1}$, P. THIBAULT $^{1}$}

I Service d'Urologie et 2 Service de Radiologie, Hôpital Tenon, Paris, France

\section{Introduction}

Une varicocèle est trouvée en moyenne chez $15 \%$ des hommes pubères, chez $35 \%$ des hommes présentant une infertilité primaire et chez $75 \%$ de ceux présentant une infertilité secondaire. Celle-ci peut rester asymptomatique ou entraîner des douleurs scrotales. Son traitement a pour but de stopper la progression des lésions testiculaires, d'améliorer la spermatogénèse et la fonction endocrine et de contrôler les douleurs par un contrôle anatomique des varicosités et échographique du reflux veineux vers les testicules. Actuellement, il existe 4 techniques chirurgicales et 1 technique radiologique, mais il n'y a pas de consensus pour définir les indications thérapeutiques : qui traiter ? quelle technique utiliser ?

\section{Matériels et méthodes}

A partir des phlébographies réalisées durant les embolisations des veines spermatiques, les auteurs discutent les arguments pour et contre chaque technique et proposent un algorythme décisionnel. Tous les patients ont reçu une information sur les risques et avantages de chaque traitement. L'embolisation est proposée en première intention. Tous les patients traités sont convoqués pour un contrôle clinique et échographique à trois mois. Si le geste initial a été insuffisant ou inefficace, un traitement chirurgical est proposé, par abord inguinal ou subinguinal associant une dissection du cordon et une libération du testicule permettant la section des veines collatérales et du gubernaculum testis.

\section{Résultats}

L'embolisation n'a jamais eu de complication majeure, seul des douleurs funiculaires ont été notées. Moins de $10 \%$ des hommes revus présentait un échec ou une insuffisance de cette première procédure et ont été opérés. La dissection du cordon était le plus souvent normale, sans varicosité, confirmant l'efficacité du traitement initial, mais il existait de larges collatérales vers le plexus de Santorini, les saphènes ou le Gubernaculum testis. Celle-ci étaient toujours visualisées sur la phlébographie initiale. Dans cette expérience, il n'y a pas eu d'atrophie testiculaire secondaire, et moins de $5 \%$ d'hydrocèle modérée. Les résultats à long terme ne peuvent être évalués car la majorité des patients ne répondent pas aux convocations. Néanmoins plusieurs cas de fécondation naturelle, après échec de FIV-ICSI, nous ont été rapportés 18 mois après ce traitement... Ces observations doivent être confirmées par des études prospectives.

\section{Conclusion}

Le premier but du traitement est de contrôler anatomiquement la varicocèle et échographiquement le reflux dans la veine spermatique. Secondairement seulement il est possible d'évaluer l'influence de ce traitement sur les paramètres spermatiques et la fertilité. L'embolisation radiologique de la veine spermatique permet ce résultat dans $90 \%$ des cas. Mais quand des veines collatérales à destinée du Santorini, des saphènes ou du gubernaculum testis sont présentes, aucune technique iliaque ne peut les contrôler. Dans notre expérience, la technique de Goldstein, qui associe une dissection du cordon à la libération du testicule par voie inguinale, permet un traitement complémentaire sûre et efficace après embolisation initiale insuffisante. 


\section{Modalités défensives utilisées dans une population d'hommes stériles : évaluation par le defense style questionnaire (DSQ), à propos de 42 patients}

\section{BELLONE1, O. COTTENCIN2, J.M. RIGOT1}

${ }^{1}$ Service d'Andrologie - Hôpital Calmette - CHRU Lille ; ${ }^{2}$ Service du Professeur M. Goudemand - Clinique de Psychiatrie - CHRU Lille

La littérature traitant de l'AMP et des troubles psychologiques et/ou sexuels associés est relativement riche. Dans la majorité des cas ces travaux ont été effectués en milieu gynécologique, avec un abord surtout féminin et conjugal du trouble. Les travaux effectués en milieu andrologique sont beaucoup plus rares. Ce constat justifie la nécessité d'explorer de nouvelles pistes pour mieux comprendre le contexte clinique qui entoure ces troubles chez l'homme. C'est dans cette perspective que nous avons entrepris une étude s'intéressant aux troubles psychologiques chez l'homme azoospermique et hypofertile et à une entité clinique récemment introduite dans la recherche psychiatrique quantitative : les mécanismes de défense. Les items du Defense Style Questionnaire (DSQ) ont été conçus pour représenter et évaluer les dérivés conscients des mécanismes mentaux de défense inconscients : les styles de défense. Cette approche évalue bien plus les mécanismes d'adaptation, les stratégies adaptatives, selon la terminologie anglosaxone, que les processus inconscients. L'objet de cette étude est de rechercher quels sont les styles de défense utilisés, d'apprécier s'il existe des modalités défensives différentes selon que l'on soit azoospermique ou hypofertile, et d'évaluer si les styles de défense adoptés peuvent contribuer à la vulnérabilité ou à la protection vis-à-vis du développement - ou pas - de troubles psychiatriques au sein d'une population andrologique.

\section{Matériels et méthodes}

42 patients ; âge moyen 32 ans ( 23 - 49 ans) ; 22 patients azoospermiques, 20 oligoasthénotératospermiques. Evalutation par le DSM IV et le DSQ par le même investigateur : Michel Bellone (psychiatre). Durée moyenne de l'entretien : 45 minutes à 60 minutes.

\section{Résultats}

Nous avons retrouvé dans notre échantillon $26.2 \%$ de troubles psychiatriques type DSM-IV avec une sur-représentation significative du trouble anxieux généralisé et du trouble somatisation par rapport à la population générale (ce qui correspond à 11 cas dépistés lors des entretiens). Notre population se défend selon des modalités défensives proches de la population générale et utilise des styles de défense appartenant préférentiellement au style dit mature. Nous y retrouvons l'humour, puis la répression et l'anticipation. La comparaison entre azoospermes et oligoasthénotératospermiques révèle qu'il n'y ni de différence significative en ce qui concerne la morbidité psychiatrique, ni en ce qui concerne l'utilisation des styles de défense. Ces deux groupes aborderaient l'infertilité de la même façon en utilisant des modalités défensives similaires. La pathologie psychiatrique dans la totalité de notre population est significativement corrélée avec l'utilisation préférentielle du retrait, de la consommation, de la formation réactionnelle et au défaut d'utilisation de l'humour.

\section{Conclusion}

S'il ne semble pas y avoir de différence de retentissement en fonction du degré d'hypofertilité, en revanche, l'existence d'une sur-représentation des troubles psychiatriques chez les patients infertiles par rapport à une population témoin impose aux andrologues de savoir entendre la souffrance au delà de la demande d'agir. Ceci justifie à notre avis que les patients doivent avoir la possibilité d'exprimer dans les unités où ils sont pris en charge tous les sentiments inhérents à leur drame personnel et conjugal.

\section{$\leftrightarrow \bullet$}

\section{Méthode simple et rapide de détection des microdé- létions du chromosome $Y$ chez les hommes infer- tiles.}

\author{
I. AKNIN-SEIFER ${ }^{1,2}, \mathrm{R}$; TOURAINE $^{2}$, H. LEJEUNE ${ }^{3}$, \\ J.L. LAURENT ${ }^{1}$, B. LAURAS ${ }^{2}$, R. LEVY ${ }^{1}$ \\ ${ }^{1}$ Laboratoire de Biologie de la Reproduction et ${ }^{2}$ Laboratoire de \\ Génétique de l'hôpital Nord, Saint-Etienne, 42055, France, \\ 3 Département de Médecine de la Reproduction, Lyon, France.
}

Introduction : Les microdélétions du chromosome Y peuvent être détectées chez les patients infertiles présentant des troubles sévères de la spermatogenèse. Ce dépistage est conseillé avant réalisation de l'ICSI. Le but de notre étude était de proposer une méthode simple et rapide de détection des microdélétions du chromosome Y. Nous avons testé la faisabilité d'un protocole d'extraction d'ADN à partir de cellules buccales en utilisant des cytobrosses et comparé deux protocoles de PCR : un protocole adapté de la technique de consensus européen et le Kit PROMEGA "Y chromosome deletion detection system 1.1".

Matériels et Méthodes : Patients : 19 patients infertiles présentant une microdélétion du chromosome $\mathrm{Y}$, préalablement diagnostiquée à l'aide d'un protocole "maison" de 15 STS (Sequence Tagged Sites), ont été inclus dans cette étude. Extraction d'ADN : L'ADN a été extrait à partir des leucocytes du sang périphérique par la technique classique du phénol chloroforme. Les cellules buccales ont été recueillies à l'aide d'écouvillons en nylon, par brossage interne des joues, puis un protocole spécifique d'extraction d'ADN à base de résines CHELEX a été appliqué. Quantification d'ADN : La quantifi- 
cation des solutions d'ADN a été réalisée par spectrophotométrie et par bandelettes colorimétriques (Invitrogen), à la fois pour l'ADN du sang périphérique et pour celui des cellules buccales. Détection des microdélétions : Les ADN ont été testés avec deux techniques de PCR différentes : une technique PCR " maison " proche des recommandations internationales de Simoni, comportant 6 marqueurs STS et 2 contrôles et les 17 marqueurs et un contrôle du Kit Promega. Les produits de PCR ont été analysés par électrophorèse en gel d'agarose.

Résultats : (1) L'extraction d'ADN à partir des cellules buccales a été possible dans tous les cas et permet d'obtenir entre 4 et $10 \mu \mathrm{g}$ d'ADN par cytobrosse. (2) Seize délétions sur les 19 échantillons d'ADN (84\%) ont été détectées par les deux méthodes. Le Kit PROMEGA n'a pas détecté deux délétions : 1/ une délétion en AZFa a échappé aux deux marqueurs du Kit supposés couvrir cette région, "sY81 et sY182". 2/ le Kit n'a pas détecté une petite délétion limitée à deux marqueurs contigus sY143 et sY142. (3) De plus, pour 4 autres patients délétés, le marqueur sY133, inclus dans le Kit donnait un signal positif faible, pouvant évoquer un résultat faussement positif. La séquence correspondant à ce locus DY223 présente de très fortes homologies avec au moins trois clones (dont gene bank AC010086) correspondant à 3 loci différents, suggérant que le STS sY133 n'est pas spécifique et conduit à de faux positifs. (4) Dans 13 autres cas, notre étude a confirmé que le marqueur sY153 du Kit correspondait à un locus polymorphique (Simoni, 1999).

Conclusion : (1) L'écouvillonnage de cellules buccales représente une alternative pratique et sure par rapport au sang périphérique pour la recherche des microdélétions du chromosome Y. (2) Il faut souligner les risques de résultats faussement positifs et faussement négatifs avec les 4 PCR multiplex du Kit PROMEGA, ainsi que les erreurs d'interprétation liées à un choix peu judicieux et mal réparti des STS. En conclusion, il est possible de proposer une technique simple, rapide et non invasive de détection des microdélétions du chromosome $\mathrm{Y}$, conforme aux recommandations internationales et nationales, permettant d'évoluer vers une standardisation des techniques.

\section{Valeur pronostique de l'inhibine B sur l'issue de la biopsie testiculaire, et sur le résultat de l'ICSI dans les azoospermies sécrétoires}

\section{BAILLY, B. GUTHAUSER, M. BERGERE, M. VERY, R. LOMBROSO, J. SELVA \\ Centre Hospitalier Poissy Saint Germain, 10 rue du Champs Gaillard, 78300 Poissy, France}

Le but de cette étude rétrospective est double : évaluer la relation existant entre la concentration en inhibine $B$ chez l'homme présentant une azoospermie sécrétoire et (1) la présence de spermatozoïdes dans le tissus testiculaire, ainsi que
(2) l'issue de l'ICSI, lorsque la biopsie était positive.

\section{Matériels et méthodes}

Patients: 72 couples présentant une azoospermie sécrétoire (confirmée par l'examen anatomo-pathologique) ont été inclus dans cette étude rétrospective. Nous avons distingué 2 groupes de patients selon les valeurs d'inhibine B: groupe $1(n=36)$, inhibine $\mathrm{B}<15 \mathrm{pg} / \mathrm{ml}$, c'est à dire présentant un taux indétectable; groupe $2(\mathrm{n}=36)$, inhibine $B \geq 15 \mathrm{pg} / \mathrm{ml}$. Une biopsie testiculaire (TESE) a été pratiquée sur ces patients, et les spermatozoïdes ont été congelés dans des microgouttes de milieu, permettant de congeler plusieurs paillettes et de réaliser plusieurs cycles d'ICSI pour une seule biopsie testiculaire.

\section{Résultats}

N. de TESE

$\%$ de TESE positive

N. de cycles d' ICSI

$\mathrm{N}$ de couples concernés

Grossesses cliniques

/ICSI (\%)

N. de grossesses évolutives

$\mathrm{N}$. de fausses couche

$\mathrm{N}$ de grossesses en cours

N. d'accouchements

N. de bébés nés

Taux de grossesse évolutive cumulé par couple

(1 à 5 tentatives d'ICSI)

\section{Groupe 1 \\ Inhibine $B<15$}

36

$19 \%(7 / 36)$

10

5

$2(20 \%)$

2

0

1

1

1

$40 \%(2 / 5)$
Groupe 2

Inhibine $B \geq 15$

36

$55 \%$

(20/36)

33

14

$8(24 \%)$

6

$2(25 \%)$

3

3

3

$42 \%$

(6/14)
Parmi les 72 biopsies réalisées, $19 \%(7 / 36)$ des biopsies étaient positives dans le groupe 1 , alors que $55 \%$ (20/36) l'étaient dans le groupe 2. Concernant l'issue des tentatives d'ICSI, les taux de grossesse sont comparables dans les deux groupes amenant à un taux de grossesse cumulé actuel par couple ayant eu au moins une tentative d'ICSI ( 1 à 5 cycles) de $40 \%$ dans le groupe 1 , à $42 \%$ dans le groupe 2 .

\section{Discussion / Conclusion}

Le pourcentage de biopsies positives est plus faible dans le groupe 1 que dans le groupe 2 . Cette étude confirme donc que le taux d'inhibine $B$ a une bonne valeur prédictive négative sur l'issue de la biopsie Toutefois dans cette étude, aucune valeur seuil d'inhibine B n'a été retrouvée en deçà de laquelle les biopsies sont toujours négatives. Toutefois, en cas d'ICSI, les chances de grossesse paraissent comparables quel que soit le dosage d'inhibine $B$. Ceci nous parait justifier de proposer une biopsie testiculaire à tous les patients présentant une azoospermie sécrétoire, après information sur leurs chances de succès. 


\section{Etude morphométrique semi-quantitative du vieillis- sement testiculaire}

L. BICCHIERAY 1 , M. ALBERT1, N. PARSEGHIAN1, M. BERGERE1, J.F. UHL2, P. MONGIAT-ARTUS3, J.P. LASSAU ${ }^{2}$, V. DELMAS 2 , O. CUSSENOT ${ }^{3}$, J. SELVA ${ }^{1}$

1 Laboratoire d'Histologie-Embryologie, UFR Biomédicale des Saints-Pères, 45 rue des Saints-Pères, 75006 Paris ; 2 Institut d'Anatomie, UFR Biomédicale des Saints-Pères, 75006 Paris ;

${ }^{3}$ EA 3104 Paris 7 (I.U.F.) 103 Bd Saint-Michel, 75005 Paris

Le don du corps du service d'Anatomie de la Faculté des Saints-Pères nous donne accès à des prélèvements de testicules d'hommes décédés à des âges variant entre 50 et 99 ans. Nous présentons ici les premiers résultats d'une étude histologique morphométrique semi-quantitative du vieillissement testiculaire sur une série de 30 sujets.

Après prélèvement autopsique des testicules dans les 24 heures suivant le décès, les investigations suivantes ont été réalisées :

Une étude macroscopique (volume, poids et densité) une étude histologique (trichrome de Masson) et une analyse morphométrique assistée par ordinateur portant sur les paramètres suivants :

- concernant les sections transversales de tubes séminifères : la surface totale, l'épaisseur de la membrane propre, la densité en noyaux..

- concernant le tissu interstitiel : la surface occupée par les cellules de Leydig, leur aspect et la densité en noyaux.

- L'étude a été complétée par un comptage de visu des différents types cellulaires de l'épithélium séminifère.

Les premiers résultats obtenus sur une série de 30 sujets âgés de 50 à 99 ans montrent plusieurs altérations en fonction de l'âge : un épaississement de l'albuginée, une sclérose des vaisseaux, une hyalinose intertubulaire, un épaississement de la membrane propre, un appauvrissement de l'épithélium séminifère, et une raréfaction associée à une hypertrophie des cellules de Leydig. L'aspect de ces dernières est étroitement corrélé avec l'état de conservation de la spermatogénèse.

Les variations individuelles sont extrêmement importantes avec de grandes altérations de la spermatogénèse dès 60 ans, avec des cellules de Leydig rares et hypertrophiées, alors qu'une spermatogénèse conservée est possible jusqu'à 89 ans avec des cellules de Leydig proches de la normale. 5 des 21 sujets âgés de plus de 60 ans avaient une spermatogénèse conservée. Ceci nous conduit actuellement à tenter de corréler ces observations à différents polymorphismes qui pourraient être impliqués dans la fertilité masculine et le contrôle hormonal de la spermatogénèse.

\section{Relations génotype-phénotype dans les microdéle- tions de la région AZFc : rôle de la suppléance par DAZL}

\section{PLOTTON1,2,3, C. DUCROS1 Y. MOREL2,}

\section{PUGEAT1,2, H. LEJEUNE1,2,3}

\author{
ILaboratoire de la Fédération d'Endocrinologie, hôpital de \\ l'Antiquaille, ${ }^{2}$ INSERM U 329, 3INSERM-INRA U 418 Hôpital \\ Debrousse, ${ }^{4}$ Département de Médecine de la Reproduction, Hôpital \\ Edouard Herriot, Lyon
}

Les microdélétions de la région $\mathrm{AZF}$ c du bras long du chromosome $\mathrm{Y}$, donnent lieu à des phénotypes variables d'azoospermie ou d'oligospermie. L'étendue de ces microdélétions ne permet pas d'expliquer les variations phénotypiques.

La région $A Z F c$ du chromosome $Y$ porte le gène $D A Z$ dans l'espèce humaine et chez les grands singes. Dans les autres espèces, la fonction de $\mathrm{DAZ}$ est assurée par le gène ancestral autosomique DAZL. Chez l'homme, une copie de ce gène autosomique DAZL persiste sur le chromosome 3.

Hypothèse : Nous avons émis l'hypothèse selon laquelle, en l'absence du gène $\mathrm{DAZ}$, emporté par une microdélétion de la région $\mathrm{AZFc}$, le gène homologue ancestral autosomique DAZL assure une suppléance Cette suppléance peut être plus ou moins efficace selon les mutations accumulées par DAZL au cours de l'évolution.

Méthodes et résultats : Nous avons séquencé la partie codante du gène DAZL chez 9 patients porteurs d'une microdélétion de la région $A Z F c$. Nous avons mis évidence les mutations suivantes : T19N ; Q28H ; L67I ; S79A T169P ; I179X ; N188H ; G253H ; A293G

Les mutations du gène DAZL sont plus nombreuses $(p<0.05)$

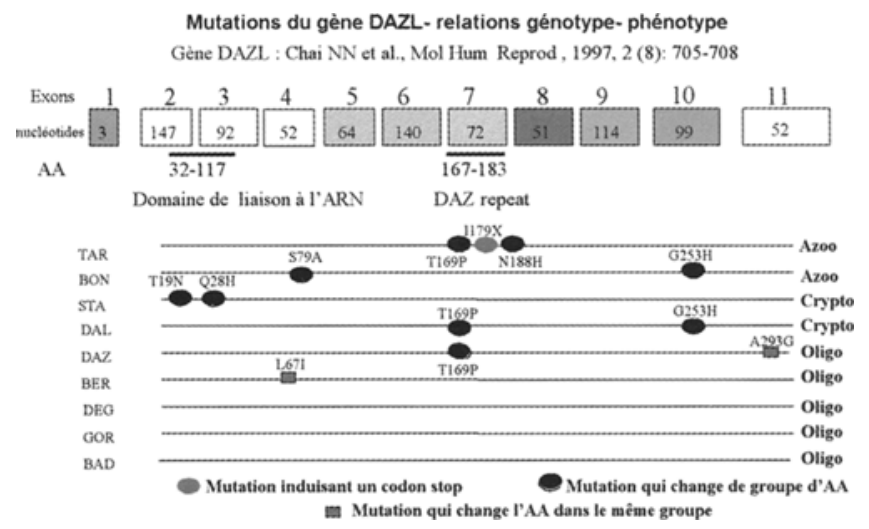

et plus sévères chez les patients présentant une azoospermie ou une cryptozoospermie $(9$ mutations pour 4 patients, un codon Stop et 8 changements de groupe d'aa) par rapport aux patients présentant une oligospermie ( 3 mutations pour 5 patients, un seul changement de groupe d'aa).

Conclusion : Ces résultats sont compatibles avec l'hypothèse d'une suppléance par le gène DAZL en cas de microdélétion de la région $\mathrm{AZFc}$ du chromosome $\mathrm{Y}$. Le phénotype paraît 
dépendre des mutations accumulées sur le gène DAZL au cours de l'évolution.

Travail financé par la Commission Recherche des Hospices Civils de Lyon.

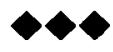

\section{Relations génotype-phénotype dans les microdélé- tions de la région AZFb : un cas d'oligospermie par réduction du nombre de copies du gène RBMY1}

\section{PLOTTON 1,2 , C. DUCROS 1 , M. PUGEAT 1 , H. LEJEUNE $1,2,3$}

ILaboratoire de la Fédération d'Endocrinologie, hôpital de l'Antiquaille, 2 INSERM-INRA U 418 Hôpital Debrousse, ${ }^{3}$ Département de Médecine de la Reproduction, Hôpital Edouard Herriot, Lyon

Les microdélétions de la région $\mathrm{AZFb}$ du chromosome $\mathrm{Y}$ sont habituellement associée à une azoospermie par blocage de la spermatogenèse au cours de la méiose. Une microdélétion de la région $\mathrm{AZFb}$ a été mise en évidence chez un patient présentant une oliogoasthénospermie, son père a la même microdélétion que lui, ainsi que son fils qui a été conçu avec un délai de 24 mois.

La région $\mathrm{AZFb}$, porte le gène RBMY1 (Ribonucleic acid Binding Motif, Y chromosome 1) présent en plusieurs copies. Il existe des pseudogènes (RBMY2) situés tout au long du chromosome $\mathrm{Y}$, un gène homologue sur le chromosome $\mathrm{X}$ (RBMX), et des homologues autosomiques (chromosome 6 et 11). Les gènes de la région $\mathrm{AZFb}$ du chromosome $\mathrm{Y}$ ont la particularité de porter une répétition de 4 exons codant pour 4 régions appelées SRGY, alors que les gènes homologues et pseudogènes ne portent qu'une seule région SRGY.

Objectifs : Notre travail a cherché à expliquer la différence de phénotype entre les microdélétions habituelles de la région $\mathrm{AZFb}$ et ce cas particulier de microdélétion transmissible.

Hypothèse : Comme la microdélétion transmissible est de plus petite taille que les microdélétions habituelles emportant l'ensemble de la région $\mathrm{AZFb}$, nous avons fait l'hypothèse d'une réduction du nombre de copies de RBMY1 pouvant expliquer ce phénotype d'oligospermie alors que les délétions de toute la région $\mathrm{AZFb}$, responsable d'azoospermie emportent toutes les copies du gène RBMY1.

Méthodes : Nous avons développé un marqueur des gènes RBMY1 de la région $\mathrm{AZFb}$ basé sur la répétition des régions SRGY.

Résultats : Ce marqueur est spécifique des gènes RBMY1 de la régions $\mathrm{AZFb}$ (il est absent en cas de microdélétion étendue responsable d'azoospermie). Il nous a permis de montrer la persistance d'un certain nombre de copies du gène RBMY1 dans la famille présentant la microdélétion transmissible. L'étude en Southern blot met en évidence un remaniement de la région $\mathrm{AZFb}$ avec réduction du nombre de copies du gène RBMY1, ce que confirme la quantification par PCR compétitive $(\mathrm{p}<0.01)$

Conclusion : Nous avons décrit une microdélétion de petite taille de la région $\mathrm{AZFb}$ associée à une oligospermie et une réduction du nombre de copies du gène RBMY1.

Travail financé par la Commission Recherche des Hospices Civils de Lyon.

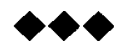

\section{Reconstruction du scrotum et du périnée après gangrène de Fournier par un lambeau pédicule inguinal et deux lambeaux en $L$}

\section{F. KIRSCH-NOIR 1 , C. CANNISTRA ${ }^{3}$, O. TRAXER 1 , G. RIGOU ${ }^{2}$, F. SAIDI ${ }^{2}$, A. PARROT ${ }^{2}$, P. THIBAULT 1}

${ }^{1}$ Service d'Urologie et ${ }^{2}$ Service de Réanimation, Hôpital Tenon, Paris, France ; ${ }^{3}$ Service de Chirurgie, Hôpital Bichat, Paris, France

\section{Introduction}

La maladie de Fournier associe un syndrome septique sévère et une cellulite d'évolution rapide. Le traitement nécessite un large débridement de tous les tissus nécrosés pour contrôler les paramètres hémodynamiques, même si une antibiothérapie ainsi que des drogues vaso-actives sont débutées parallèlement. L'oxygéno-thérapie hyper bare peut également être proposée. A distance, après détersion et bourgeonnement, le choix du meilleur protocole de reconstruction sera discuté. Cicatrisation dirigée, greffe de peau totale ou en filet, lambeaux pédiculés ont été utilisés en fonction de la localisation, de la surface de la perte de substance, mais aussi de l'expérience et des habitudes de l'opérateur.

\section{Matériel et méthode}

M. T, 58 ans a subit l'exérèse du scrotum et du périnée, en deux temps, pour contrôler une gangrène de Fournier. Les deux testicules ont été placés, dans leur vaginale, dans des logettes sous cutanées inguinales. L'état hémodynamique instable du patient n'a pas permis l'oxygénation hyper-bare. Une dérivation sus-pubienne des urines et une colostomie ont été réalisés ainsi qu'une trachéotomie. Pendant un mois de réanimation, la détersion et le pansement au miel ont été réalisés tous les jours. La reconstruction a été réalisée, d'abord par un lambeau inguinal qui a permis de reconstruire le scrotum et ensuite par deux lambeaux en L qui ont couvert le périnée et les fosses ischio-rectales. Le lambeau inguinal de $19 \mathrm{~cm}$ de long a été prélevé en regard de l'artère circonflexe iliaque superficielle, parallèlement au ligament iliaque, limité en haut 
à $2 \mathrm{~cm}$ et en bas à $6 \mathrm{~cm}$ de celui-ci. La dissection à permis de libérer le pédicule vasculo-nerveux et de tubuler la peau autour de lui pour le préserver, avant de tourner le lambeau sur cet axe et de couvrir les testicules. Après 4 semaines, le pédicule à été libéré et la région péri-anale couverte par deux lambeaux en $\mathrm{L}$ Toutes les stomies ont été refermées à 4 mois.

\section{Résultats}

L'examen microscopique des lésions oedématiés, mais non nécrosées, réséqués 2 heures après la première intervention, pour contrôler le sepsis, montrait des lésions de vascularite intense avec accumulation de germes anaérobies et de polynucléaires autour des capillaires. L'examen clinique à 3 ans montre un bon résultat esthétique et fonctionnel. La trophicité et la sensibilité du néo-scrotum et des testicules sont normales. L'érection, l'éjaculation, la miction et la défécation sont normales.

\section{Conclusion}

Une large exérèse de tous les tissus pathologiques (oedématiés et nécrosés) doit être réalisée très rapidement et parallèlement à une réanimation poussée pour contrôler le sepsis. La surveillance rapprochée du site opératoire ou en cas de mauvais contrôle hémodynamique, permet d'élargir secondairement l'exérèse si nécessaire. A distance, l'utilisation de lambeaux pédiculés, notamment pour la reconstruction de zones de plis et de frottement, permet d'obtenir une couverture trophique et sensible qui évite les dysesthésies, sensation de peau sèche et les érosions rencontrées lors de l'utilisation de greffe de peau ou les brides et cicatrices rétractiles observées après cicatrisation dirigée.

\section{La plicature de l'albuginée dans le traitement du recurvatum penis secondaire à la maladies de Lapeyronie: notre expérience}

\section{G. PASSAVANTI, V. PIZZUTI, A. BRAGAGLIA, R. PAOLINI \\ Service d'Urologie-Andrologie Hopital "Misericordia" Grosseto (Italie)}

Une petite déviation du pénis est trouvée très souvent dans la maladie de LaPeyronie (IPP) et seulement $10 \%$ des malades ont la nécessité d'une intervention chirurgicale corrective.

26 malades, d'age compris entre 51 et 76 années, atteints par une IPP avec un Recurvatum Pénis (RP) important $\left(>35^{\circ}\right)$ ont été suivis pendant une période de 31-64 mois (moyenne 45,5 mois). Tous les malades referaient une trouble importante de la pénétration. Les 14 premiers malades ont eu une pharmacocavernosometrie-graphie (PCMG), pour préciser les mécanismes veno-occlusifs et pour avoir une documentation eidologique très précise; les 12 suivante ont eu une hétéro-photographie durant une érection pharmaco-induite. Cette dernière méthode diagnostique est moins précise mais et aussi moins invasive. La plaque fibreuse a été étudiée cliniquement et par échographie. Le RP était dorsal dans 3 cas $(11.5 \%)$, ventral dans 2 cas ( $7.6 \%)$, dorso-latéral dt dans 14 cas $(53.8 \%)$ et dorso-latérale gauche dans 7 cas $(26.9 \%)$. Nous avons toujours exécuté une libération du faisceau vasculo-nerveux dorsal et du corps spongieux, en correspondance du sommet de la courbure. Chez 14 malades nous avons utilisé le point de Klevmark en prolene $2 / 0$, et, chez 12 malades nous avons placé une série de 3 petits points en prolene 3/0, juxtaposés, parallèles, longs 6 $\mathrm{mm}$, disposés long l'axe majeur du pénis et séparés $1-2 \mathrm{~mm}$ l'un de l'autre (Essed modifié). Dans 2 cas de RP très importante nous avons coupée long l'axe transversale et donc suturée long l'axe longitudinale la plaque fibreuse, pour réduire la tension sur les points de plicature. Après avoir vérifié la correction du RP, nous avons fermé le F. de Buck, le dartos et la peau après postectomie.

Dans la période postopératoire nous avons relevé un oedème de la peau du pénis et une hypo-orgasmie pendant 3-6 mois. Durant la suite nous avons interrogé les malades sur leur satisfaction sur le plan fonctionnel et esthétique. 6 malades ont présenté un déficit érectile d'une façon progressive et ils ont utilisées injections intracaverneusese PGE1. Dans une période de 3-5 ans, 5 (19.2\%) malades ont présenté une progression de la plaque avec une reprise du RP, et 3 patients avaient une trouble de la pénétration. Les sujets opérés selon Klevmark avaient des petits nodules (bien tolérés) au niveau des points de plicature, tandis que les 12 opérés selon Essed (modifié) ne les avaient pas. Le pénis se raccourcit au maximum de $2 \mathrm{~cm} .23$ malades ont manifesté la satisfaction des partenaires.

Plusieurs techniques ont été proposées pour traiter le RP, elles s'inspirent à deux principes :

-Allonger la partie convexe de la courbure enlevant la plaque ( substituée par une paroi veineuse, une greffe de goretex etc..)

-raccourcir la partie convexe par dissection d'une ellipse d'albuginée, ou par des points de plicature.

Dans notre expérience la plicature de l'albuginée a donné des bons résultats soit dans la période postopératoire, soit pour la satisfaction à long terme, esthétique et fonctionnelle, des malades ( $80 \%$ des cas); la progression de la plaque a causé une récidive du RP depuis longtemps (3-5 années) dans le 19.2\% des cas; d'ailleurs la maladie de Lapeyronie a une évolution imprévisible mais l'évolution très ralentie conseille toujours la chirurgie du RP importante. Avoir utilisé des points $3 / 0$ en série n'a pas déterminé la présence de nodules sur le profil du pénis (dérangement relevé avec d'autres techniques).

En conclusion nous pouvons faire de réflexions empiriques mais à notre avis intéressantes: une bonne libération des corps caverneux permet de mieux placer les points lesquels doivent traverser à tout épaisseur l'albuginée; l'utilisation de fil 3/0 en série réduit le dérangement déterminé par des nodules sur le profil du pénis et enfin, le point de plicature est réversible en 
cas d'hypercorrection. A notre avis, donc, la plicature de l'albuginée représente une bonne technique pour traiter le RP secondaire à la maladie de Lapyronie.

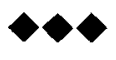

\section{Facteurs pronostiques d'aggravation dans les oli- gospermies sévères}

\author{
J. PERRIN, J.C. DELAROZIÈRE, J. SAÏAS-MAGNAN, \\ O. PAULMYER-LACROIX, C. METZLER-GUILLEMAIN, \\ L. LACROIX-SERTHELON, G. PORCU-BUISSON, \\ F. CARLES-MARCORELLE, M.R. GUICHAOUA, \\ J.M. GRILLO
}

Laboratoire de Biologie de la Reproduction, Hôpital la Conception, 145 bd Baille, 13385 Marseille cedex 5

But de l'étude : cette étude a pour but de rechercher des critères permettant de prévoir une aggravation des paramètres spermatiques chez les patients présentant une oligospermie sévère (numération des spermatozoïdes $<5$ Millions $/ \mathrm{ml}$ de sperme).

Matériel et Méthodes : étude rétrospective portant sur 69 patients consultant pour infertilité, ayant pratiqué au moins 3 spermogrammes dans notre Unité de Spermiologie et présentant une oligospermie sévère.

Analyse statistique : la population étudiée a été divisée en 2 groupes :

- oligospermies sévères s'aggravant significativement : diminution du nombre de spermatozoïdes/ml d'un facteur $\geq 2$ entre le premier et le dernier spermogramme du patient.

- oligospermies sévères stables : variation de la numération des spermatozoïdes inférieure à un facteur 2 entre le premier et le dernier spermogramme.

Cette étude a comparé entre ces deux groupes les différents paramètres du spermogramme initial : numération par $\mathrm{ml}$, numération par éjaculât, \% de mobilité, \% de spermatozoïdes progressifs, \% de formes typiques, vitalité, ainsi que l'âge du patient et son taux de FSH.

\section{Résultats :}

$\begin{array}{llll}\text { Vitalité } & \text { Aggravation } & \text { Stabilité } & \\ \leq 50 \% & 8(31 \%) & 2(6 \%) & 10(17 \%) \\ 50-70 \% & 13(50 \%) & 16(52 \%) & 29(51 \%) \\ >70 \% & 5(19 \%) & 13(42 \%) & 18(32 \%) \\ & 26 & 31 & \mathbf{p}=\mathbf{0 . 0 2 8}\end{array}$

Nous mettons en évidence une diminution significative de la vitalité des spermatozoïdes dans le groupe "aggravation" par rapport au groupe "stabilité".
Les taux de FSH plasmatique, la mobilité totale, la mobilité progressive, la cytologie des spermatozoïdes ne diffèrent pas significativement dans les deux groupes.

Discussion : de nombreuses ICSI sont réalisées dans des cas d'oligospermie majeure, où le risque d'un recueil inexploitable le jour de la PMA n'est pas négligeable et peut nécessiter une ponction chirurgicale "de secours". La pratique d'une auto-conservation de spermatozoïdes systématique chez tous ces patients n'étant pas envisageable, il apparaît souhaitable de dégager des facteurs permettant de prévoir une aggravation des paramètres spermatiques compromettant la réalisation de l'ICSI. Notre étude met en évidence une vitalité significativement plus faible dans les spermes évoluant vers l'aggravation ; les taux de FSH plasmatique des patients présentant une aggravation y semblent plus élevés que dans le groupe des oligospermies sévères stables. Ces résultats préliminaires seront affinés par une étude à plus grande échelle. A terme, la présence de facteur(s) prédictif(s) d'aggravation chez un patient présentant une oligospermie sévère pourrait justifier la réalisation immédiate d'une auto-conservation, en évitant ainsi un prélèvement chirurgical de spermatozoïdes ultérieur.

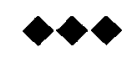

\section{Les blastocystes sont indicateurs de bons résultats cliniques de I'ICSI avec sperme testiculaire}

\section{VIRANT-KLUN, T. TOMAZEVIC, S. DROBNIC, I. BACER-KERMAVNER, J. MIVSEK, H. MEDENVRTOVEC}

Service de gynecologie-obstetrique, Centre clinique universitaire, Slajmerjeva 3, 1000-Ljubljana Slovenie

\section{Introduction}

Récemment, l'introduction en fécondation in vitro de nouveaux milieux séquentiels permet la culture d'embryons jusqu'au stade blastocyste. Cette étude évalue le développement des blastocystes issus d'ICSI utilisant les spermatozoïdes testiculaires et le rôle clinique chez les azoospermiques des embryons pluricellulaires (5ème jour) par rapport aux embryons à faible cellularité (2ème jour).

\section{Matériel et méthodes}

Après ICSI avec sperme testiculaire, les embryons ont été cultivés 5 jours jusqu'au stade blastocyste dans les milieux séquentiels M1 et M2 (Medicult, Danemark). Nous avons évalué la proportion d'embryons qui atteignaient le stade blastocyste et les résultats cliniques (taux de grossesse et d'avortement) obtenus après transfert de blastocystes (5ème jour) ou d'embryons à faible cellularité (2ème jour).

\section{Résultats}

Au 5ème jour il y avait plus d'embryons non-avancés $(<10$ 
cellules) après ICSI avec sperme testiculaire qu'après FIV classique ( $56 \%$ vs $15 \%, P<0.005)$ ou après ICSI avec sperme éjaculé ( $56 \%$ vs $30 \%, P<0.05$ ). Au 5 ème jour, on transférait 2 embryons - blastocystes, morulae, ou des embryons nonavancés. Le taux général de grossesses par transtert d'embryons au 5ème jour était le même que celui obtenu par transfert au 2ème jour d'embryons à faible cellularité (18\% vs $18 \%$ ). On n'observait pas plus de différence en ce qui concerne le taux d'avortement (33\% vs $25 \%$ ) tandis que-dans les deux groupes le taux d'avortement était supérieur à celui obtenu après ICSI avec sperme éjaculé $(11 \%, P<0.05)$ ou après FIV classique pour indications féminines $(9 \%, P<0.05)$. Après utilisation de blastocystes, on observait un fort taux de grossesse (36\%) sans avortement spontané (0\%), tandis qu'avec les embryons qui n'atteignaient pas le stade blastocyste au 5ème jour, moins de grossesses étaient obtenues (morulae : $21 \%$ et embryons non-avancés : $5 \%, P<0.05$ ) et plus de grossesses s'achevaient en avortement spontané (morulae: $67 \%$ et embryons non avancés: $100 \% ; P<0.005$ ).

\section{Conclusion}

Chez les azoospermiques, les effets paternels peuvent être délétères sur le développement embryonnaire. Au 5èrne jour, seuls les blastocystes devraient être transférés. Les morulae et les embryons non-avancés sont l'indication à un diagnostic préimplantatoire. La culture prolongée d'embryons serait un outil important pour éviter les risques génétiques chez ces patients.

\section{La prévalence d'ureaplasma urealyticum à titre élevé est-elle augmentée dans le sperme en cas d'infertilité inexpliquée?}

\footnotetext{
F. VIRECOULON 1 , F. WALLET 2 , M.C. PEERS 1 , A. DEFOSSEZ1, R. COURCOL ${ }^{2}$, V. MITCHELL1

ILaboratoire de Spermiologie/Histologie-Embryologie \& Biologie de la Reproduction et 2 Laboratoire de Bactériologie-Hygiène, Hôpital Calmette, CHRU, 59037 Lille
}

Introduction. Le rôle d'Ureaplasma urealyticum (UU) sur la fertilité est controversé. Malgré son implication initiale dans l'infertilité inexpliquée, aucun impact d'UU n'a été démontré par la suite sur de plus larges populations. Il semble pourtant qu'il pourrait affecter le transport des spermatozoïdes à travers le tractus génital féminin, avoir une action négative sur le taux de grossesse et augmenter le taux d'avortement après FIV. Cependant, l'espèce est commensale, ce qui implique de ne pas considérer simplement son absence ou sa présence, mais de quantifier cette présence, un titre $\geq 10^{4} \mathrm{UCC} / \mathrm{ml}$ étant reconnu comme pathogène.

Matériel et méthode. 132 couples ont été sélectionnés, pour lesquels les paramètres spermatiques et le test post-coïtal sont normaux. Le bilan féminin a comporté un suivi des courbes de température, une hystérosalpingographie complétée éventuellement d'une coelioscopie, et une exploration endocrinienne poussée. L'infertilité a alors été classée selon la cause principale en ovarienne, tubaire, sur endométriose et inexpliquée. Les infertilités cervico-utérines, trop peu représentées, ont été éliminées.

Après recueil de sperme respectant une asepsie optimale et transport rapide au laboratoire de Bactériologie, UU a été titré en utilisant le kit Mycoplasma Duo® (Bio-Rad), permettant une semi-quantification en : absence/ titre faible $\left(\leq 10^{3}\right.$ $\mathrm{UCC} / \mathrm{ml}) /$ titre fort $\left(\geq 10^{4} \mathrm{UCC} / \mathrm{ml}\right)$.

\section{Résultats}

voir tableau

\section{Conclusion}

Quel que soit le titre d'UU, il n'y a pas d'association préférentielle à un type donné d'infertilité. En particulier, l'incidence d'UU n'est pas augmentée dans l'infertilité inexpliquée.

La bactérie proliférant en compagnie d'autres espèces, en particulier Gardnerella vaginalis, il importe à présent de rechercher si l'association d'UU à une autre espèce est surreprésentée dans les infertilités inexpliquées.
UU

\begin{tabular}{lllll} 
Infertilités & absence & $\begin{array}{c}\text { UU } \\
\text { titre faible } \\
\left(\leq \mathbf{1 0}^{\mathbf{3}} \mathbf{U C C} / \mathbf{m l}\right)\end{array}$ & $\begin{array}{l}\text { titre fort } \\
\left(\geq \mathbf{1 0}^{\mathbf{4}} \mathrm{UCC} / \mathrm{ml}\right)\end{array}$ & $\mathbf{3 7}$ \\
\hline ovarienne & $\mathbf{3 1}(83,8 \%)$ & $\mathbf{3}(8,1 \%)$ & $\mathbf{3}(8,1 \%)$ & $\mathbf{4 1}$ \\
tubaire & $\mathbf{3 4}(82,9 \%)$ & $\mathbf{6}(14,6 \%)$ & $\mathbf{1}(2,4 \%)$ & $\mathbf{1 8}$ \\
sur endométriose & $\mathbf{1 5}(83,3 \%)$ & $\mathbf{0}(0 \%)$ & $\mathbf{3}(16,7 \%)$ & $\mathbf{3 6}$ \\
inexpliquée & $\mathbf{2 8}(77,8 \%)$ & $\mathbf{5}(13,9 \%)$ & $\mathbf{3}(8,3 \%)$ & $\mathbf{1 3 2}$ \\
total & $\mathbf{1 0 8}$ & $\mathbf{1 4}$ & $\mathbf{1 0}$ & \\
\hline
\end{tabular}

